\title{
ATP site ligands determine the assembly state of the Abelson kinase regulatory core via the activation loop conformation
}

\author{
Rajesh Sonti ${ }^{1}$, Ines Hertel-Hering ${ }^{1}$, Allan Joaquim Lamontanara ${ }^{2}$, Oliver Hantschel $^{2}$, \& \\ Stephan Grzesiek ${ }^{1}$
}

${ }^{1}$ Focal Area Structural Biology and Biophysics, Biozentrum, University of Basel, CH-4056 Basel, Switzerland

${ }^{2}$ Swiss Institute for Experimental Cancer Research (ISREC), School of Life Sciences, École polytechnique fédérale de Lausanne (EPFL), 1015 Lausanne, Switzerland

*Address correspondence to:

Stephan Grzesiek

Focal Area Structural Biology and Biophysics, Biozentrum

University of Basel, CH-4056 Basel, Switzerland

Phone: ++41612672100

FAX: $\quad++41612672109$

Email: Stephan.Grzesiek@unibas.ch

Keywords: cancer, chronic myelogenous leukemia, allosteric regulation, structure, dynamics, NMR 


\section{ABSTRACT}

The constituent SH3, SH2, and kinase domains of the Abl kinase regulatory core can adopt an assembled (inactive) or a disassembled (active) conformation. We show that this assembly state strictly correlates with the conformation of the kinase activation loop induced by a total of 14 ATP site ligands, comprising all FDA-approved Bcr-Abl inhibiting drugs. The disassembly of the core by certain ligands can be explained by an induced push on the kinase N-lobe via A- and P-loop towards the SH3 domain. A similar sized P-loop motion is expected during nucleotide binding and release, which would be impeded in the assembled state, in agreement with its strongly reduced kinase activity.

\section{INTRODUCTION}

Abelson tyrosine kinase (Abl) plays a crucial role in many cellular processes such as proliferation, division, survival, DNA repair, migration and is an important cancer drug target. $^{1-3}$ The regulatory core of Abl (Figure 1a) consists sequentially of the N-cap, the SH3 and SH2 domains, and the kinase domain (KD) N- and C-lobes, which are separated by the catalytic cleft. ${ }^{4}$ This SH3-SH2-KD domain order is conserved among the Abl, Src, Csk, Brk, and Tec non-receptor tyrosine kinase families. Under physiological conditions, Abl is autoinhibited by interactions of the SH3 and SH2 domains with the KD N- and C-lobes, respectively, as well as by the docking of the myristoylated N-terminal glycine-2 (present in the Abl $1 \mathrm{~b}$ splice variant) into a hydrophobic cavity of the KD C-lobe. ${ }^{4,5}$ This 'assembled' state of the $\mathrm{Abl}$ core is observed in crystal structures (Figure 1a) ${ }^{4,5}$ as well as in solution ${ }^{6,7}$ in the presence and absence of myristoylation. Similar assembled core structures have so far been observed for the $\mathrm{Src}^{8}$ and $\mathrm{Btk}^{9}$ kinases.

Upon activation, the SH3 and SH2 domains disengage from the KD and are able to bind to cellular partners. ${ }^{2}$ The high activity of this 'disassembled' state may be explained by the readiness to form such intermolecular interactions, but also intramolecular interactions are involved. In particular, interactions between the Abl SH2 domain and the KD N-lobe have a critical role in leukemogenesis ${ }^{10}$ and enhance in vitro kinase activity ${ }^{11}$. Corresponding direct contacts between the SH2 domain and the top of the KD N-lobe have been observed under ${\text { certain } \text { crystal }^{4,10,12} \text { and solution }}^{11,13}$ conditions, which may explain the enhanced activity by a modulation of the hinge motion between $\mathrm{N}$ - and C-lobes. ${ }^{14,15}$

Abnormal reciprocal translocation between chromosomes 9 and 22 results in the deregulated fusion protein Bcr-Abl and subsequently in chronic myelogenous leukemia $(\mathrm{CML}) .{ }^{16}$ The ATP site inhibitors imatinib (Gleevec), nilotinib (Tasigna), and dasatinib (Sprycel) represent the front-line therapy against CML. ${ }^{17-19}$ However, spontaneous point 
mutations render these inhibitors ineffective in a fraction of patients. ${ }^{18}$ In particular, the multidrug-resistant "gatekeeper" T334I mutation (Abl 1b numbering) has stimulated the search for new therapeutics. ${ }^{20,21}$ Among those are the ATP site inhibitors ponatinib (Iclusig) ${ }^{20}$ and axitinib, ${ }^{22}$ as well as the allosteric inhibitor asciminib ${ }^{23}$ targeting the myristoyl binding pocket. The presumed mode of action of the latter is a stabilization of the autoinhibited, assembled state. ${ }^{7,24}$ Besides their efficacy against CML, inhibitors of Bcr-Abl are also widely used against Bcr-Abl-expressing subsets of B-cell leukemias, several diseases with Kit and Platelet-derived growth factor receptor (PDGF-R) aberrations, ${ }^{25}$ and have promising effects in cell and animal models of Parkinson's disease. ${ }^{26,27}$

Several structural motifs of the Abl KD are conserved in most kinases and crucial for the catalytic function (Figure 1c): the activation loop (A-loop) with the Asp-Phe-Gly (DFG) motif, the $\alpha \mathrm{C}$ helix, and the glycine-rich (phosphate-binding loop) P-loop. ${ }^{28,29}$ The A-loop adopts two major conformations observed in most ligand complexes (Figure 1c,d and Figure S1) ${ }^{18}$ For type I inhibitors, the A-loop is in the 'active' (open) conformation positioned towards the $\alpha \mathrm{C}$ helix (e.g. in the dasatinib complex) ${ }^{30}$ with the aspartate of the DFG oriented 'in' or 'flipped' towards the ATP binding pocket. In contrast, type II inhibitors induce the 'inactive' (closed) conformation of the A-loop, where the entire loop is rotated towards the Ploop (e.g. imatinib complex) and the aspartate oriented 'out' from the ATP pocket. ${ }^{29}$ In this conformation, the central part of the A-loop mimics the bound substrate. ${ }^{31}$ Both the $\alpha \mathrm{C}$ helix position $^{12,28}$ and the P-loop conformation ${ }^{32}$ vary depending on the A-loop conformation and the interaction with the ligand.

NMR RDC data on the Abl KD prove that in solution the A-loop predominantly ( $>85 \%)$ adopts the same active conformation in a type I (dasatinib) complex and the same inactive conformation in type II (imatinib and nilotinib) complexes as observed in respective crystal structures. ${ }^{33}$ However, backbone ${ }^{15} \mathrm{~N}$ NMR relaxation data also indicate dynamics of the Aloop in the nanosecond and microsecond regime, corresponding to exchange with minor conformations on the order of a few percent. Surprisingly, solution NMR and SAXS data on a larger Abl construct comprising the SH3-SH2-KD domains of the regulatory core show unequivocally that the core is in the assembled (autoinhibited) state in its apo form, but disassembles when the inhibitors imatinib or nilotinib bind (Figure 1b). ${ }^{7}$ As evident from ${ }^{15} \mathrm{~N}$ relaxation data, this disassembled, but inhibited state is characterized by a high nanosecond mobility of the SH3 and SH2 domains relative to the KD. So far, no crystal structures of such complexes have been obtained. The addition of the allosteric, myristoyl binding pocket inhibitor GNF5 to complexes of the Abl core with imatinib or nilotinib restores the assembled 
state. ${ }^{7}$ Thus the allosteric inhibitor stabilizes the assembled state relative to the disassembled state induced by imatinib or nilotinib.

Since imatinib and nilotinib induce the inactive A-loop conformation and at the same time disassemble the core, we hypothesized an allosteric coupling between the A-loop and the $\mathrm{KD}: \mathrm{SH} 3-\mathrm{SH} 2$ interface and systematically investigated the conformations of the Abl regulatory core in complex with a total of 14 ATP site binding ligands. The results show that the inactive A-loop conformation induced by type II inhibitors leads to the disassembly of the core, whereas ATP site ligands with active A-loop conformations preserve the assembled core. An analysis of all available Abl crystal structures reveals that the type II inhibitors induce a small but consistently observed push of the KD N-lobe via the A-loop and the P-loop towards the SH3 domain. This push apparently destabilizes the KD:SH3-SH2 interface and causes the disassembly of the core. A mechanic model is proposed by which allosteric myristoyl binding pocket inhibitors relieve the exerted strain on the KD:SH3-SH2 interface by bending the $\alpha$ I' helix and keeping the core in the assembled conformation. The detected allosteric link between A-loop, P-loop and core assembly state provides atomic details on the autoinhibition mechanism of the assembled state, since the hindrance of P-loop motions by the SH3 domain in the assembled state is expected to severely inhibit ATP binding and ADP release.

\section{RESULTS AND DISCUSSION}

\section{The active and inactive A-loop conformations induced by type I and II inhibitors correlate strictly with the assembled and disassembled state of the regulatory core}

To carry out a comprehensive study of the assembled and disassembled state of the SH3SH2-KD Abl regulatory core (residues 83-534) in complex with ATP site ligands, we have obtained all commercially available ligands, for which Abl complex structures have been deposited in the PDB. This set of 14 ligands comprises all 5 FDA-approved Bcr-Abl inhibiting drugs, several drug candidates that are or were in clinical trials, as well as the ATP analogue AMP-PNP (Figure S2). The conformational state of the Abl regulatory core under solution conditions can be determined from characteristic NMR ${ }^{1} \mathrm{H}_{-}{ }^{15} \mathrm{~N}$ backbone chemical shifts of residues within the SH3 and SH2 domains. ${ }^{7}$ Figure 2a shows such characteristic shifts for residues V130, T136, G149 and V170 in the apo form and in complexes with the various ATP site ligands. As exemplified for V130, dasatinib, bosutinib, and AMP-PNP induce similar shifts as the apo form, corresponding to an assembled state, whereas imatinib, nilotinib, and ponatinib complexes exhibit shifted resonances corresponding to the disassembled state.

A quantitative analysis of all the investigated complexes was carried out on all ${ }^{1} \mathrm{H}-{ }^{15} \mathrm{~N}$ chemical shifts from the $\mathrm{SH} 3$ and $\mathrm{SH} 2$ domains. Chemical shift deviations $\Delta \delta$ relative to the apo form are shown in Figure $\mathbf{2 b}$. Large deviations (Figure $\mathbf{2 b}$, left) at many sites indicate the 
disassembled state for imatinib, nilotinib, ponatinib, bafetinib, and rebastinib, whereas for all other cases (Figure 2b, right) only small deviations are observed corresponding to the assembled state. A numerical classification of these ${ }^{1} \mathrm{H}-{ }^{15} \mathrm{~N}$ chemical shifts by Principal Component Analysis (PCA) yields a clear distinction between the assembled and disassembled conformations via the first principal component PC1 (Figure 2c, PC1 and PC2 explain $84 \%$ of the data).

We then asked whether PC1 correlates also to the A-loop conformation observed in various Abl complex structures. For this, the PDB was searched for entries with $\geq 90 \%$ sequence similarity to the human Abl imatinib complex (2HYY) yielding a total of 51 structures (Supplementary Table 1). No correlations are evident in these structures between the A-loop conformation and the crystallographic space groups or B-factors (Supplementary Table 1). However with no exception (Figure 2e), all type I ligand (Figure S2a) complexes with the characteristic active A-loop conformation ( $\mathrm{C}^{\alpha}$ distance $\mathrm{G} 409-\mathrm{K} 375, \mathrm{~d}_{409 / 375} \approx 12 \AA$ ) show chemical shifts corresponding to the assembled state $(\mathrm{PC} 1 \approx-0.1)$. In contrast, all type II ligand complexes (Figure S2b) with the inactive A-loop conformations $\left(\mathrm{d}_{409 / 375} \approx 32 \AA\right)$ induce the disassembled state $(\mathrm{PC} 1 \approx 0.2)$. Thus the A-loop conformation is directly correlated to the assembly state of the multidomain Abl core.

Interestingly within the assembled conformations, PC2 further distinguishes the apo form and the AMP-PNP complex from the type I inhibitors (Figure 2C). An inspection of the corresponding chemical shift changes of the $\mathrm{SH} 2$ and $\mathrm{SH} 3$ domains indicates slight conformational rearrangements at their interfaces to the $\mathrm{KD} \mathrm{N}$ - and C-lobes as well as at their mutual contact sites (Figure S3). Thus, type I inhibitors induce long-range interactions towards the $\mathrm{SH} 2$ and $\mathrm{SH} 3$ domain, which are different from those of the non-inhibited apo form and the AMP-PNP complex.

\section{The mechanism of core disassembly induced by type II inhibitors}

To identify the mechanism by which the inactive A-loop conformation induces the core disassembly, we aligned all $51 \mathrm{Abl}$ structures on the KD C-lobe (Figure 3a). In all complex structures of KD-only constructs with type II ligands (inactive A-loop conformations, magenta), the KD N-lobe is slightly rotated by $\sim 5-10^{\circ}$ relative to KD complexes with type I ligands (active A-loop conformations, green) in the direction of the SH3 domain found in the full $\mathrm{Abl}$ regulatory core structures. The center of the rotation is located close to residue G340 in the hinge between $\mathrm{N}$ - and C-lobe and the rotations induce $\sim 2-5 \AA$ displacements of residues facing the SH3 domain (e.g. K282) (Figure 3b). The reason for this movement of the N-lobe can be traced to steric interactions between the inhibitor, the A-loop and the P-loop (Figure 
3c). As described previously, ${ }^{30}$ a steric clash between type II ligands and residues F401 and S404 prevents the A-loop from adopting the active conformation (green) and induces the inactive conformation (magenta). A-loop residues F401 and M407 in this inactive conformation would clash with residues Q271 and Y272 of the P-loop in the active conformation. As a result, the P-loop is pushed by 1-2 $\AA$ upward in the direction of the KD Nterminus (e.g. V275 in Figure 3b) resulting in the overall 5-10 rotation of the N-lobe towards the SH3 domain. This conformation is further stabilized by $\pi-\pi$ interactions between the ligand and the Y272, Y336 and F382 side chains ${ }^{32}$ as well as ion-pair interactions between A-loop residue R405 and residues E298/E301 at the N-terminal end of the $\alpha \mathrm{C}$ helix (Figure S4a). In contrast, the active A-loop conformation is stabilized by a salt bridge between residue R405 and residue $\mathrm{E} 311$ at the $\mathrm{C}$-terminal end of the $\alpha \mathrm{C}$ helix as well as two $\mathrm{H}$-bonds between $\mathrm{A}$ loop residue S404 and residue I379 in the KD C-lobe (Figure S4b,c).

In all solved structures of the complete assembled core, which comprise three independently deposited ternary complexes with PD166326 (type I) and myristic acid as well as one ternary complex with nilotinib (type II) and asciminib (Figure 3a,b yellow/orange and Figure S5), the N-lobe is positioned between the two conformations found for type I and type II ligand complexes of KD-only structures. Apparently, the presence of the $\mathrm{SH} 3$ domain confines the N-lobe to this intermediate position. Nevertheless, in the type II nilotinib/asciminib core structure, the N-lobe is rotated by about $5^{\circ}$ more towards the $\mathrm{SH} 3$ domain than in the type I PD166326/myristic acid core structures.

\section{A mechanical model of allosteric effects controlling core assembly}

All observations on the allosteric effects of ATP site and myristoyl binding pocket ligands onto the assembly state of the core can be rationalized by a simple mechanical model (Figure 3d). In complexes of KD-only constructs with type II inhibitors the A-loop is pushed to the inactive conformation resulting in the rotation of the KD-N lobe towards the SH3 interface. In such constructs and in the absence of a suitable ligand in the myristoyl pocket, the C-terminal $\alpha \mathrm{I}$ ' helix of the KD-C lobe is flexible in solution as evident from NMR. ${ }^{7,34}$ In $\mathrm{KD}$ crystal structures without myristoyl binding site ligands, this helix adopts a straight conformation, which would clash with the SH2 domain in the assembled core. ${ }^{5}$ We assume that albeit being flexible, helix $\alpha$ I' also samples this clashing conformation to some extent in solution. The full Abl core in its apo form is assembled in solution and its KD-N lobe shows micro- to millisecond mobility as evidenced by NMR line broadening. ${ }^{7}$ In this state, the motion of $\alpha$ I' must be restricted due to the steric hindrance by the $\mathrm{SH} 2$ domain. This results in a reduction of entropy and a higher free energy, i.e. a force directed, but not yet sufficient, to open the core. 
Complexes of the core with type I inhibitors have very similar conformations as the assembled apo core. However, it is unclear whether type I inhibitors change the dynamical behavior of the KD N-lobe since not many residues could be observed in this region due to low NMR signal intensity. In complexes of the core with type II inhibitors, the KD N-lobe rigidifies ${ }^{7}$ and pushes towards the SH3 domain. This force acts synergistically with the force exerted by the flexible $\alpha$ I' helix onto the SH2 domain and overcomes the forces holding the assembled core together. As a result, the core snaps open in a similar way as releasing a spring latch. Finally, in ternary complexes of the core with type II inhibitors and myristoyl binding pocket ligands, which bend helix $\alpha \mathrm{I}$ ' away from the SH2 domain, the opening force is reduced and the core stays assembled. $^{7}$

For the clinical response to kinase inhibitors, besides molecular binding mode and its influence on the assembly state of the Abl core domains that are described here, additional mechanisms will certainly also contribute. These include the presence of Bcr-Abl tetramers in cells due to the $\mathrm{N}$-terminal coiled-coil oligomerization domain ${ }^{35}$ and a yet to be explored possible overall structural difference due to different chromosomal translocation breakpoints that give rise to the two major Bcr-Abl protein isoforms p210 and p190. As recently demonstrated p210 and p190 have substantially different protein interactors and downstream signaling networks in leukemia cells ${ }^{36}$, which result in different kinase inhibitor sensitivities and possibly different clinical responses.

\section{Relating core assembly, A-loop/P-loop conformation and autoinhibition of kinase activity}

The role of the SH3 domain and the core assembly for Abl autoinhibition has been demonstrated in pioneering cellular assays where interactions between the SH3 domain and the linker to the KD had been abrogated by specific point mutations resulting in strongly enhanced kinase activity. ${ }^{37}$ Activity assays carried out on the SH3-SH2-KD, SH2-KD (residues 138-534) and a KD-only ${ }^{11}$ construct using the peptide substrate Abltide reproduce this strong autoinhibiting effect of the SH3 domain (Figure S6): e.g. the Michaelis-Menten $\mathrm{V}_{\max }$ of the SH3-SH2-KD construct (assembled core in apo form) is about 4-fold (3-fold) reduced relative to the $\mathrm{SH} 2-\mathrm{KD}$ (KD-only) construct.

The observed correlation between A-/P-loop conformation and the core assembly state gives new insight into this autoinhibition mechanism at the atomic scale. In complex structures of the $\operatorname{Irk}^{38}$ and $\mathrm{Abl}$ (Figure 3e $)^{28}$ kinases with ATP analogues and peptide substrates, the Aloop is in the active, open conformation, whereas the peptide substrate fills a similar position as part of the A-loop in the inactive, closed conformation. ${ }^{31}$ In these ternary complexes, the Ploop tightly encloses the ATP molecule from the side of the N-lobe thereby fixing this part of 
the catalytic center. This results in an N-lobe orientation, which is very similar to the ones observed in type I inhibitor complexes, but distinct from the type II complexes, where the hinge between N- and C-lobe is slightly more open (Figure 3e). It has been noted early on that in this conformation nucleotide entry or release must be strongly inhibited and that the opening of the catalytic cleft by a rotation of the N-lobe is the likely prerequisite for ADP release, which is the rate limiting step in many kinases. ${ }^{39}$ We can estimate from the solved ternary complexes that, in order to allow nucleotide binding or release, the P-loop must move by at least one atom layer (Figure 3e) in the direction of the SH3 domain of the fully assembled core. This movement will be very similar in size and direction as the one observed for the change between the active (type I inhibitor) and inactive (type II inhibitor) A-loop structures, e.g. a movement of P-loop residue V275 by $1.5 \AA$ (van der Waals radius of oxygen) will induce a $7^{\circ}$-rotation of the entire N-lobe around the hinge (G340) in the direction of the SH3 interface. This motion is possible in the disassembled regulatory core, but must be strongly hindered in the assembled core by the presence of the SH3 domain thereby reducing the speed of ATP binding and ADP release and hindering the catalytic reaction (Figure $\mathbf{3 f}$ ).

\section{CONCLUSION}

In summary, we have shown a strict correlation between the A-loop conformation induced by ATP-binding pocket ligands and the overall assembly state of Abl's regulatory core. All type I ligands, which leave the A-loop in the open, active state, keep the core assembled as in the apo conformation. In contrast, all type II ligands, which push the A-loop into the closed, inactive conformation, disassemble the core. The core opening can be rationalized by steric forces from the ligand via the A-loop and P-loop onto the kinase N-lobe towards the SH3 domain. A simple mechanical model connects this mechanism to the stabilization of the assembled core by allosteric myristoyl binding pocket inhibitors, which reduce the pressure onto the KD:SH3-SH2 interface by bending helix $\alpha I^{\prime}$.

The tight connection between A-/P-loop and core assembly state also provides highresolution insight into the autoinhibition mechanism, since it implies that the SH3 domain in the assembled state severely hinders the P-loop motion, which would be required for nucleotide binding and release. An identical inhibition mechanism is expected for all nonreceptor tyrosine kinases with an $\mathrm{SH}-\mathrm{SH} 2-\mathrm{KD}$ core.

The autoinhibition by the assembled core constitutes only one of Abl's regulation mechanisms. Interactions with cellular partners and phosphorylation provide further layers of regulation. In particular upon $\mathrm{Abl}$ activation, the activation loop gets phosphorylated at Y412, ${ }^{40.41}$ which stabilizes the open, active A-loop conformation..$^{22}$ A second phosphorylation 
site, Y245, is located in the SH2-kinase linker. Phosphorylation of Y245 prevents assembly of the core ${ }^{2}$ and strongly enhances trans-phosphorylation of Y412 by a second Abl molecule, ${ }^{11}$ whereas its mutation impairs Abl activation. ${ }^{40}$ The binding of type II inhibitors induces a disassembled, yet inhibited state. We have previously shown that in $\mathrm{Ba} / \mathrm{F} 3$ cells, phosphorylation of residue Y245 in the SH2-KD linker ${ }^{7}$ increases for type II inhibitor concentrations close to $\mathrm{IC}_{50}$, presumably due to the disassembly of the core. This may serve as a priming mechanism for enhanced Abl activity after inhibitor washout, although no increased downstream signaling has been detected in cellular experiments. ${ }^{43}$ Nevertheless, the effects of inhibitor-induced Abl core opening should be carefully considered in all in vivo situations, since they are universal for all type II inhibitors. In particular, emerging Abl mutants with lower affinity for type II inhibitors will still undergo core opening in the bound form, which in combination with the changed on- or off-rates may alter the dynamic equilibrium of $\mathrm{Abl}$ regulation and subsequent cellular reactions, thereby providing routes to resistance. 


\section{Figure legends}

Figure 1. Conformations of the Abl regulatory core. (a) Crystal structure of the Abl regulatory core in complex with PD166326 and myristic acid (PDB ID: 2FO0). The SH3 domain (blue), SH2 domain (yellow), SH2-kinase linker (red), A-loop (green), P-loop (green), KD N-lobe and KD C-lobe (KD-N, KD-C, sand) are shown as cartoon, the chemical structures of PD166326 and myristic acid as sticks. (b) One of the possible conformations derived from NMR and SAXS data of the Abl•imatinib complex. ${ }^{7}$ Both SH2 and SH3 domains are mobile on the nanosecond time scale (indicated by light blue arrows). Same color scheme as in a, except for the P- and A-loops, which are shown as magenta. (c) Superposition of KD crystal structures of imatinib (sand) and dasatinib (gray) complexes (PDB ID: 2HYY and 2GQG) shown as cartoons. A-loop and P-loop are shown as magenta in the imatinib and as green in the dasatinib complexes. Chemical structures of imatinib and dasatinib are shown as sticks (d) A-loop conformations in active and inactive orientations with the three conformations of the DFG motif out, in and flip. The PDB codes of the structures are indicated in Supplementary Table 1. The color code corresponds to the one of Figure 2c.

Figure 2. Evidence of allosteric coupling between A-loop conformation and assembly state of the Abl core. (a) Individual ${ }^{1} \mathrm{H}_{-}{ }^{15} \mathrm{~N}$ TROSY resonances of selected residues of the Abl core which show characteristic shifts for the assembled and disassembled state. Resonances are color-coded according to the different ATP site ligands indicated on the left. The positions of the residues are indicted as spheres within the Abl core structure (cartoon). (b) Averaged ${ }^{1} \mathrm{H}$ and ${ }^{15} \mathrm{~N}$ chemical shift differences $\Delta \delta=\left(\Delta \delta_{\mathrm{N}}{ }^{2} / 25+\Delta \delta_{\mathrm{HN}}\right)^{1 / 2}$ between the ATP site ligand complexes and the apo form of the Abl core in the region of the SH3 and SH2 domains (residues 83-234). The disassembled and assembled complexes are shown on the left and right side, respectively. (c) The first two principal components (PC1 and PC2) of the SH3 and $\mathrm{SH} 2$ domain chemical shifts of the various Abl core complexes and the apo form derived from PCA. Assembled and disassembled conformations cluster in two regions. (d) Schematic illustration of the assembled and disassembled core and the respective A-loop conformations. The distance $\mathrm{d}_{409 / 375}$ between the $\mathrm{C}^{\alpha}$ atoms of G409 and K375 (solid spheres) is taken as an indication of the active and inactive A-loop conformation. (e) Correlation between PC1 and $\mathrm{d}_{409 / 375}$. The electron density for the G409 atom was absent in the crystal structures of the ponatinib and bafetinib complexes. However, adjacent residues indicate an inactive A-loop also for these complexes. 
Figure 3. Mechanism of allosteric interaction regulating the conformations and activity of the Abl core. (a) Superposition of a representative subset of Abl crystal structures aligned on the KD C-lobe: KD-only structures with inactive A-loop conformations (type II inhibitors, see Supplementary Table 1, magenta), KD-only structures with active A-loop conformations (type I inhibitors, see Supplementary Table 1, green), SH3-SH2-KD structure (5MO4) in complex with the type II inhibitor nilotinib and the allosteric inhibitor asciminib (orange). For the latter, the $\mathrm{C}^{\alpha}$ atoms of T259, V275, K282, E294, and E327 are indicated as spheres. (b) Positions of the $\mathrm{C}^{\alpha}$ atoms of T259, V275, K282, E294, and E327 for all investigated 51 structures (Supplementary Table 1) shown within their plane of closest distance. The colorcoding follows panel (a). The positions for three further SH3-SH2-KD structures in complex with the type I inhibitor PD166326 and myristic acid (1OPK, 1OPL, 2FO0) are depicted in yellow. (c) Superposition of A-loop and P-loop conformations in KD crystal structures of imatinib (2HYY, magenta) and dasatinib (2GQG, green). Regions of steric clashes are represented as dotted spheres on the imatinib structure. (d) Mechanical model explaining the allosteric coupling between A-loop and P-loop conformation, the flexibility of the $\alpha$ I' helix, and the Abl core assembly state. Different assembly states are induced by type I (green) and II (magenta) inhibitors of the ATP-binding site and of allosteric myristoyl binding pocket inhibitors (light blue). Light blue arrows indicate mobile elements, red bars mechanical forces. (e) Structural evidence that nucleotide binding and release requires KD hinge opening. Left: KD structure in complex with ATP analogue and substrate (2G2F). A space-filling representation of the active site region is shown on the top. Right: superposition of KD complexes with ATP analogue and substrate $(2 \mathrm{G} 2 \mathrm{~F}$, blue) and with imatinib (1OPJ, red). The rotation of the N-lobe towards the SH3 interface is clearly visible for the imatinib complex, whereas for the ATP analogue-substrate complex the N-lobe has a similar orientation as in type I inhibitor complexes (see panel a). (f) model depiction of hinge motion required for nucleotide binding and release. The hinge motion is possible in the disassembled core, but strongly hindered in the assembled core. 


\section{EXPERIMENTAL SECTION}

Ligands. All ligands were purchased from commercial suppliers and used without further purification (Supplementary Table 2). Bosutinib was confirmed to be the correct isomer by solution NMR. ${ }^{44}$

Protein expression and purification. The 83-534 fragment of Abl (Abl $1 \mathrm{~b}$ numbering) was expressed in ${ }^{15} \mathrm{~N}$-labeled form in E. coli strain BL21(DE3) and purified as described earlier ${ }^{7}$ with a slight modification. After the ion exchange purification step with a Q-sepharose HP column (GE Healthcare), fractions containing Abl were pooled to a typical volume of $20 \mathrm{ml}$ and about $10 \mu \mathrm{M}$ protein concentration. To this $500 \mu \mathrm{l}$ of $12 \mu \mathrm{M}$ of Lambda protein phosphatase (LPP) were added and the final buffer conditions adjusted to $2 \mathrm{mM}$ TCEP, 100 $\mathrm{mM} \mathrm{NaCl}, 1 \mathrm{mM} \mathrm{MnCl}, 0.01 \%$ Brij $35,50 \mathrm{mM}$ Tris $\cdot \mathrm{HCl} \mathrm{pH}$ 8. After incubation at $4^{\circ} \mathrm{C}$ overnight, ESI-MS analysis showed a complete de-phosphorylation of the protein sample. All experiments were carried in this de-phosphorylated form, which significantly enhanced the stability of the NMR samples to several days even for the least stable apo state. The protein was then subjected to gel filtration on a 16/600 Superdex 200 column (GE Healthcare) for the final purification step as before.

The 138-536 fragment of SH2-KD Abl (Abl 1b numbering) construct was expressed in $E$. coli strain BL21(DE3) and purified using the published protocol. ${ }^{11}$

The plasmid 42934 containing pPET-PKR/PPase for Lambda protein phosphatase (LPP) was purchased from Addgene. PKR was removed by restriction digestion with NdeI and EcoRI resulting in a pPET-PPase. Bacterial cultures were grown in E. coli strain BL21(DE3) in $\mathrm{LB}$ medium at $25^{\circ} \mathrm{C}$ to an $\mathrm{OD}_{600}$ of 0.6 and the protein expression was induced by addition of $200 \mu \mathrm{M}$ isopropyl- $\beta$-D-thiogalactopyranoside. The cultures were pelleted down after $16 \mathrm{~h}$, and the cells were resuspended in lysis buffer [50 mM Tris $\cdot \mathrm{HCl}(\mathrm{pH}$ 7.8), $2 \mathrm{mM}$ EDTA, $20 \%$ (vol/vol) glycerol] and lysed using a M-110L microfluidiser (Microfluidics) operating at $12,000 \mathrm{psi}$. Lysates were cleared by centrifugation at $30,000 \mathrm{~g}$. The supernatant was loaded on a Q-sepharose HP column (GE Healthcare) that was pre-equilibrated with buffer A [50 mM Tris $\cdot \mathrm{HCl} \mathrm{pH}$ 7.8, $2 \mathrm{mM}$ EDTA]. The protein was eluted with a linear gradient of $0-100 \%$ buffer B [50 mM Tris $\cdot \mathrm{HCl}$ pH 7.8, $2 \mathrm{mM}$ EDTA, $200 \mathrm{mM} \mathrm{NaCl}$ ] in $6 \mathrm{CV}$ (column volume). Fractions containing LPP were pooled and adjusted to a final concentration of $500 \mathrm{mM} \mathrm{NaCl}$. The solution was then applied to phenylsepharose column (GE Healthcare) and washed with buffer A [50mM Tris $\cdot \mathrm{HCl} \mathrm{pH} 7.8,500 \mathrm{mM} \mathrm{NaCl}, 2 \mathrm{mM}$ EDTA] followed by buffer B [50mM 
Tris $\cdot \mathrm{HCl} \mathrm{pH}$ 7.8, $2 \mathrm{mM}$ EDTA, $50 \%$ (vol/vol) glycerol]. The flow through containing LPP was concentrated and subjected to gel filtration on a 26/60 Superdex 75 column (GE Healthcare) equilibrated with $50 \mathrm{mM}$ Tris $\cdot \mathrm{HCl} \mathrm{pH} 7.8,0.1 \mathrm{mM}$ EDTA, $10 \%$ (vol/vol) glycerol. All purification steps were performed at $4{ }^{\circ} \mathrm{C}$. Fractions containing LPP were then put into a storage buffer [50mM Tris $\cdot \mathrm{HCl} \mathrm{pH}$ 7.8, $100 \mathrm{mM} \mathrm{NaCl}, 2 \mathrm{mM}$ DTT, $0.01 \%$ Brij35, $100 \mathrm{mM}$ EDTA, $100 \mathrm{mM} \mathrm{MnCl}_{2}, 50 \%$ (vol/vol) glycerol], snap frozen with liquid nitrogen and stored at $-80{ }^{\circ} \mathrm{C}$ for further use.

Mass Spectrometry. The purified protein samples were applied to a Jupiter $(5 \mu, 300 \AA$, $157 \mu$ l) $\mathrm{C} 4$ reverse phase column (Phenomenex). The phosphorylation state of the protein were determined by ESI-TOF mass spectrometry (Bruker microTOF) using maximum entropy deconvolution.

NMR Spectroscopy. As in our previous study ${ }^{7}$, the isotope-labeled SH3-SH2-KD Ab1 construct was typically concentrated to $\sim 100 \mu \mathrm{M}$ and $270 \mu \mathrm{l}$ volume in $20 \mathrm{mM}$ Tris $\cdot \mathrm{HCl}(\mathrm{pH}$ 8.0), $100 \mathrm{mM} \mathrm{NaCl}, 2 \mathrm{mM}$ EDTA, $2 \mathrm{mM}$ TCEP, and $0.02 \% \mathrm{NaN}_{3}$. Ligands pre-dissolved in DMSO were added in the ratio of 3:1 (ligand: protein). The used slightly higher $\mathrm{pH}$ relative to crystallographic studies on this construct (PDB entries 2FO0, 5MO4: $\mathrm{pH}$ 7.5; PDB entries 1OPL, 1OPK: $\mathrm{pH}$ 7.0) provided higher stability in solution, presumably due to the larger offset from its pI 5.9. All NMR experiments were performed at $303 \mathrm{~K}$ on a Bruker AVANCE 900$\mathrm{MHz}$ spectrometer equipped with a TCI triple resonance cryoprobe. ${ }^{1} \mathrm{H}^{-15} \mathrm{~N}$ TROSY experiments were recorded with $224\left({ }^{15} \mathrm{~N}\right) \times 1024\left({ }^{1} \mathrm{H}\right)$ complex points and acquisition times of $\sim 40 \mathrm{~ms}$ in both ${ }^{15} \mathrm{~N}$ and ${ }^{1} \mathrm{H}$ dimensions. All data were processed with NMRPipe software package $^{45}$ and analyzed with SPARKY. ${ }^{46}$ The principal component analysis of chemical shift variations was performed using MATLAB (Mathworks).

Analysis of PDB structures. PDB structures were analyzed and displayed using the PyMOL Molecular Graphics System (Schrodinger, LLC)

\section{Supporting Information}

The Supporting Information is available free of charge on the ACS Publications website at DOI:XXX

Supplementary Figures 1-6 showing further structural and enzymatic analyses of Abl inhibitor interactions (PDF)

Supplementary Table 1-2 giving statistics of analyzed Abl crystal structures and commercial suppliers of inhibitors (XSLX) 


\section{Acknowledgements}

This work was supported by the Swiss Cancer League (grants KFS-3603-02-2015 to R.S. and S.G., KLS-3132-02-2013 and KLS-3595-02-2015 to A.J.L. and O.H.) and the Swiss National Science Foundation (grants 31-149927 and 31-173089 to S.G.). L. Skora, R. Jacob, J. Schlotte, C. Opitz, and H.J. Sass are gratefully acknowledged for helpful discussions. 


\section{References}

(1) Greuber, E. K.; Smith-Pearson, P.; Wang, J.; Pendergast, A. M. Nat. Rev. Cancer 2013, 13, 559.

(2) Hantschel, O.; Superti-Furga, G. Nat. Rev. Mol. Cell Biol. 2004, 5, 33.

(3) Hantschel, O. Genes Cancer 2012, 3, 436.

(4) Nagar, B.; Hantschel, O.; Young, M. A.; Scheffzek, K.; Veach, D.; Bornmann, W.; Clarkson, B.; Superti-Furga, G.; Kuriyan, J. Cell 2003, 112, 859.

(5) Hantschel, O.; Nagar, B.; Guettler, S.; Kretzschmar, J.; Dorey, K.; Kuriyan, J.; Superti-Furga, G. Cell 2003, 112, 845.

(6) Nagar, B.; Hantschel, O.; Seeliger, M.; Davies, J. M.; Weis, W. I.; Superti-Furga, G.; Kuriyan, J. Mol. Cell. 2006, 21, 787.

(7) Skora, L.; Mestan, J.; Fabbro, D.; Jahnke, W.; Grzesiek, S. Proc. Natl. Acad. Sci. USA 2013, 110, 4437.

(8) Xu, W.; Harrison, S. C.; Eck, M. J. Nature 1997, 385, 595.

(9) Wang, Q.; Vogan, E. M.; Nocka, L. M.; Rosen, C. E.; Zorn, J. A.; Harrison, S. C.; Kuriyan, J. eLife Sciences 2015, 4, e06074.

(10) Sherbenou, D. W.; Hantschel, O.; Kaupe, I.; Willis, S.; Bumm, T.; Turaga, L. P.; Lange, T.; Dao, K. H.; Press, R. D.; Druker, B. J.; Superti-Furga, G.; Deininger, M. W. Blood 2010, 116, 3278.

(11) Lamontanara, A. J.; Georgeon, S.; Tria, G.; Svergun, D. I.; Hantschel, O. Nat. Commun. 2014, 5, 5470.

(12) Lorenz, S.; Deng, P.; Hantschel, O.; Superti-Furga, G.; Kuriyan, J. Biochem. J. 2015, $468,283$.

(13) Saleh, T.; Rossi, P.; Kalodimos, C. G. Nat. Struct. Mol. Biol. 2017, 4, e06074.

(14) Dölker, N.; Górna, M. W.; Sutto, L.; Torralba, A. S.; Superti-Furga, G.; Gervasio, F. L. PLoS Comput. Biol. 2014, 10, e1003863 EP.

(15) La Sala, G.; Riccardi, L.; Gaspari, R.; Cavalli, A.; Hantschel, O.; De Vivo, M. J. Chem. Theory Comput. 2016, 12, 5563.

(16) Laurent, E.; Talpaz, M.; Kantarjian, H.; Kurzrock, R. Cancer Res. 2001, 61, 2343.

(17) Shah, N. P. Science 2004, 305, 399.

(18) Hantschel, O.; Grebien, F.; Superti-Furga, G. Cancer Res. 2012, 72, 4890.

(19) O'Hare, T.; O'Hare, T. Cancer Res. 2016, 76, 5911.

(20) Senior, M. Nat. Biotechnol. 2014, 32, 9.

(21) Mobitz, H.; Jahnke, W.; Cowan-Jacob, S. W. Curr Top Med Chem 2017, 17, 59.

(22) Pemovska, T.; Johnson, E.; Kontro, M.; Repasky, G. A.; Chen, J.; Wells, P.; Cronin, C. N.; McTigue, M.; Kallioniemi, O.; Porkka, K.; Murray, B. W.; Wennerberg, K. Nature 2015, 519, 102.

(23) Wylie, A. A.; Schoepfer, J.; Jahnke, W.; Cowan-Jacob, S. W.; Loo, A.; Furet, P.; Marzinzik, A. L.; Pellé, X.; Donovan, J.; Zhu, W.; Buonamici, S.; Hassan, A. Q.; Lombardo, F.; Iyer, V.; Palmer, M.; Berellini, G.; Dodd, S.; Thohan, S.; Bitter, H.; Branford, S.; Ross, D. M.; Hughes, T. P.; Petruzzelli, L.; Vanasse, K. G.; Warmuth, M.; Hofmann, F.; Keen, N. J.; Sellers, W. R. Nature 2017, 543, 733.

(24) Zhang, J.; Adrián, F. J.; Jahnke, W.; Cowan-Jacob, S. W.; Li, A. G.; Iacob, R. E.; Sim, T.; Powers, J.; Dierks, C.; Sun, F.; Guo, G.-R.; Ding, Q.; Okram, B.; Choi, Y.;

Wojciechowski, A.; Deng, X.; Liu, G.; Fendrich, G.; Strauss, A.; Vajpai, N.; Grzesiek, S.; Tuntland, T.; Liu, Y.; Bursulaya, B.; Azam, M.; Manley, P. W.; Engen, J. R.; Daley, G. Q.; Warmuth, M.; Gray, N. S. Nature 2010, 463, 501.

(25) Corless, C. L.; Barnett, C. M.; Heinrich, M. C. Nat. Rev. Cancer 2011, 11, nrc3143.

(26) Lindholm, D.; Pham, D. D.; Cascone, A.; Eriksson, O.; Wennerberg, K.; Saarma, M.

Front. Aging Neurosci. 2016, 8, 254.

(27) Pagan, F.; Hebron, M.; Valadez, E. H.; Torres-Yaghi, Y.; Huang, X.; Mills, R. R.; 
Wilmarth, B. M.; Howard, H.; Dunn, C.; Carlson, A.; Lawler, A.; Rogers, S. L.;

Falconer, R. A.; Ahn, J.; Li, Z.; Moussa, C. J. Parkinsons dis. 2016, 6, 503.

(28) Levinson, N. M.; Kuchment, O.; Shen, K.; Young, M. A.; Koldobskiy, M.; Karplus, M.; Cole, P. A.; Kuriyan, J. PLoS Biol. 2006, 4, e144.

(29) Cowan-Jacob, S. W.; Fendrich, G.; Floersheimer, A.; Furet, P.; Liebetanz, J.; Rummel, G.; Rheinberger, P.; Centeleghe, M.; Fabbro, D.; Manley, P. W. Acta Crystallogr. D Biol. Crystallogr. 2007, 63, 80.

(30) Tokarski, J. S.; Newitt, J. A.; Chang, C. Y. J.; Cheng, J. D.; Wittekind, M.; Kiefer, S. E.; Kish, K.; Lee, F. Y. F.; Borzillerri, R.; Lombardo, L. J.; Xie, D.; Zhang, Y.; Klei, H. E. Cancer Res. 2006, 66, 5790.

(31) Schindler, T.; Bornmann, W.; Pellicena, P.; Miller, W. T.; Clarkson, B.; Kuriyan, J. Science 2000, 289, 1938.

(32) Wang, Q.; Zorn, J. A.; Kuriyan, J. Meth. Enzymol. 2014, 548, 23.

(33) Vajpai, N.; Strauss, A.; Fendrich, G.; Cowan-Jacob, S. W.; Manley, P. W.; Grzesiek, S.; Jahnke, W. J. Biol. Chem. 2008, 283, 18292.

(34) Jahnke, W.; Grotzfeld, R. M.; Pellé, X.; Strauss, A.; Fendrich, G.; Cowan-Jacob, S. W.; Cotesta, S.; Fabbro, D.; Furet, P.; Mestan, J.; Marzinzik, A. L. J. Am. Chem. Soc. 2010, 132, 7043.

(35) Zhao, X.; Ghaffari, S.; Lodish, H.; Malashkevich, V. N.; Kim, P. S. Nat. Struct. Mol. Biol. 2002, 9, 117.

(36) Reckel, S.; Hamelin, R.; Georgeon, S.; Armand, F.; Jolliet, Q.; Chiappe, D.; Moniatte, M.; Hantschel, O. Leukemia 2017 31:7 2017, 31, 1502.

(37) Barilá, D.; Superti-Furga, G. Nat. Genet. 1998, 18, 280.

(38) Hubbard, S. R. EMBO J. 1997, 16, 5572.

(39) Cox, S.; Radzio-Andzelm, E.; Taylor, S. S. Curr. Opin. Struct. Biol. 1994, 4, 893.

(40) Brasher, B. B.; Van Etten, R. A. J. Biol. Chem. 2000, 275, 35631.

(41) Dorey, K.; Engen, J. R.; Kretzschmar, J.; Wilm, M.; Neubauer, G.; Schindler, T.; Superti-Furga, G. Oncogene 2001, 20, 8075.

(42) Young, M. A.; Shah, N. P.; Chao, L. H.; Seeliger, M.; Milanov, Z. V.; Biggs, W. H.; Treiber, D. K.; Patel, H. K.; Zarrinkar, P. P.; Lockhart, D. J.; Sawyers, C. L.; Kuriyan, J. Cancer Res. 2006, 66, 1007.

(43) Skora, L.; Kempf, D.; Mestan, J.; D'Orazio, D.; Jahnke, W. Eur. J. Haematol. 2015, n. (44) Boxer, S. G. PLoS ONE 2012, 7, e29828.

(45) Delaglio, F.; Grzesiek, S.; Vuister, G. W.; Zhu, G.; Pfeifer, J.; Bax, A. J. Biomol. NMR 1995, 6, 277.

(46) Goddard, T. D.; Kneller, D. G. SPARKY, version 3; University of California, 2008. 
a

b
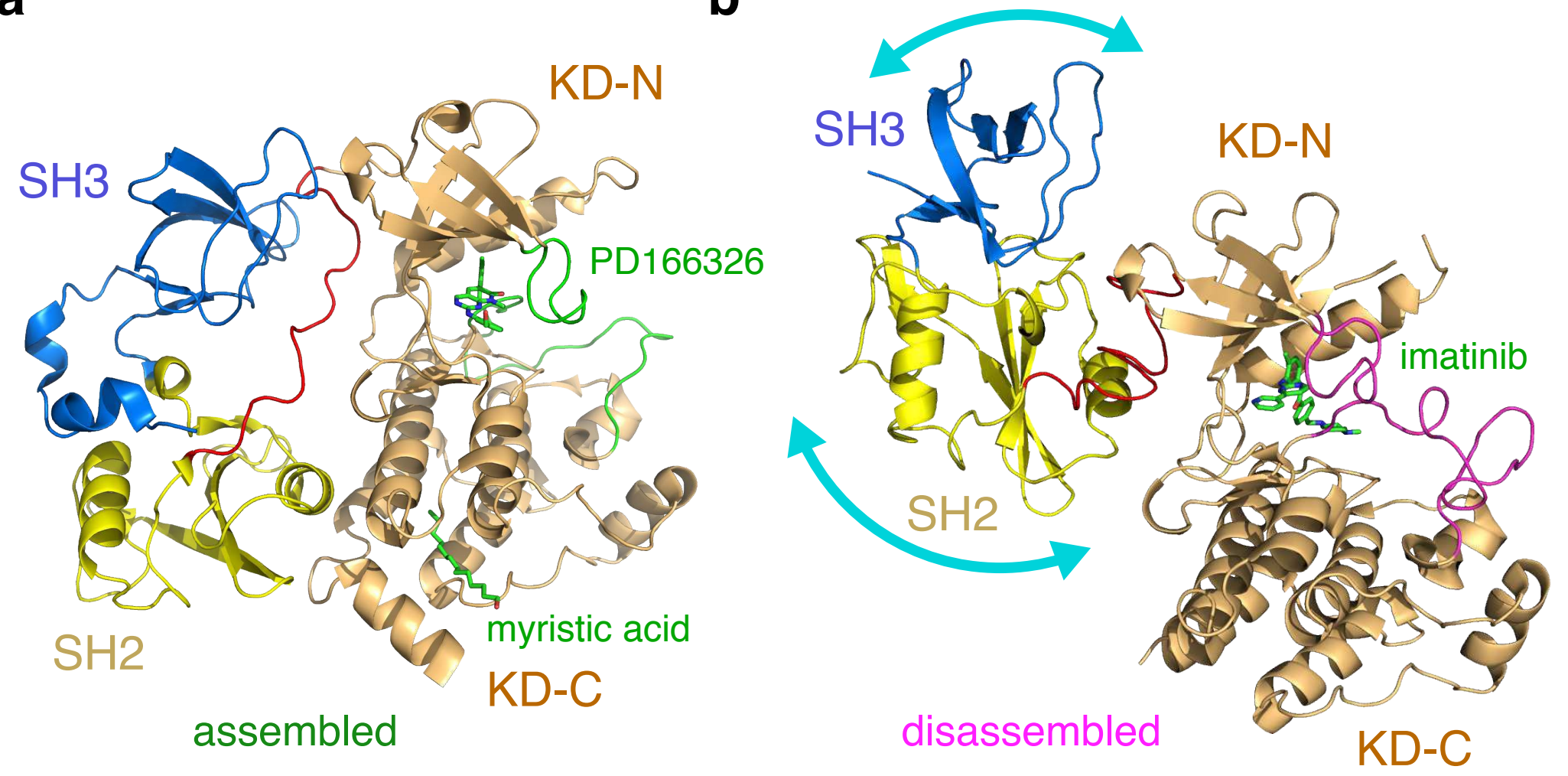

d

C

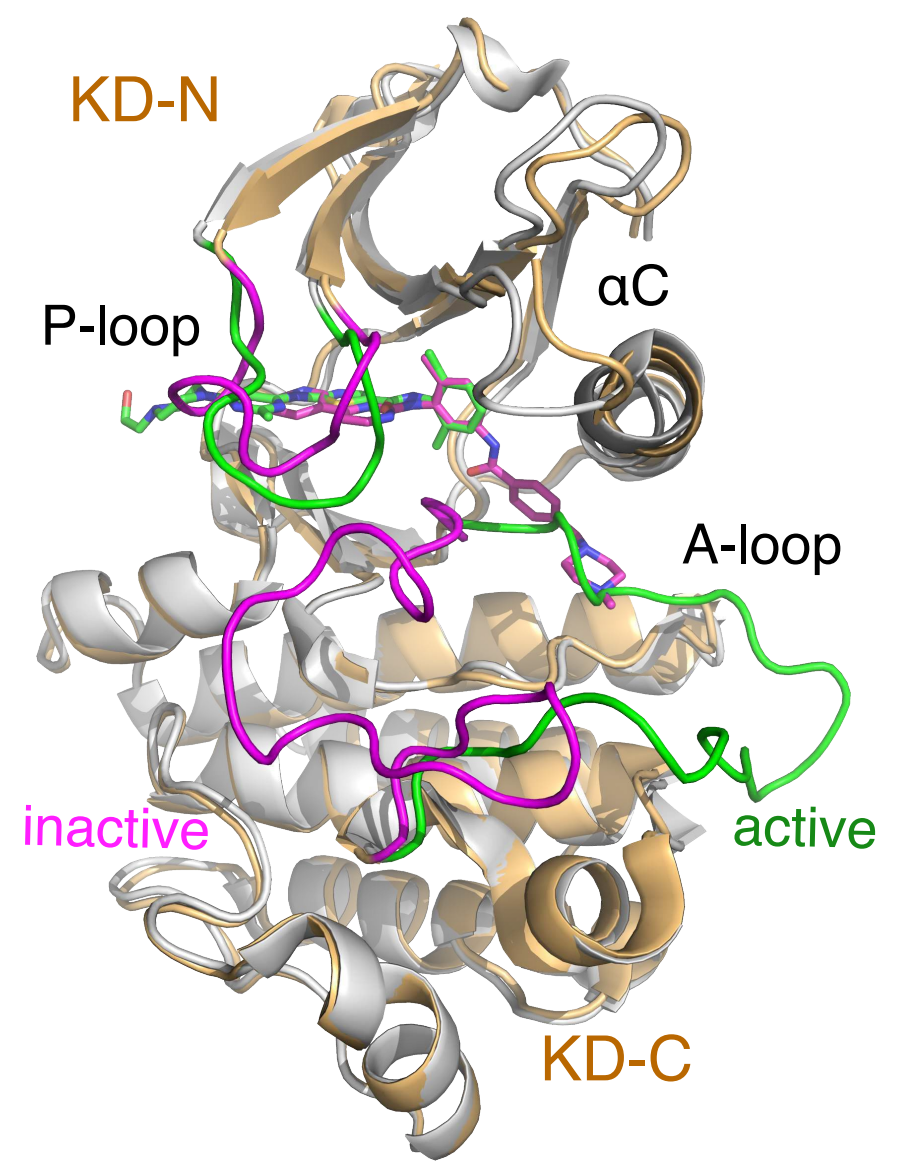

myristic acid

KD-C

assembled

$\mathrm{SH} 2$

Fig 1. Sonti et al.

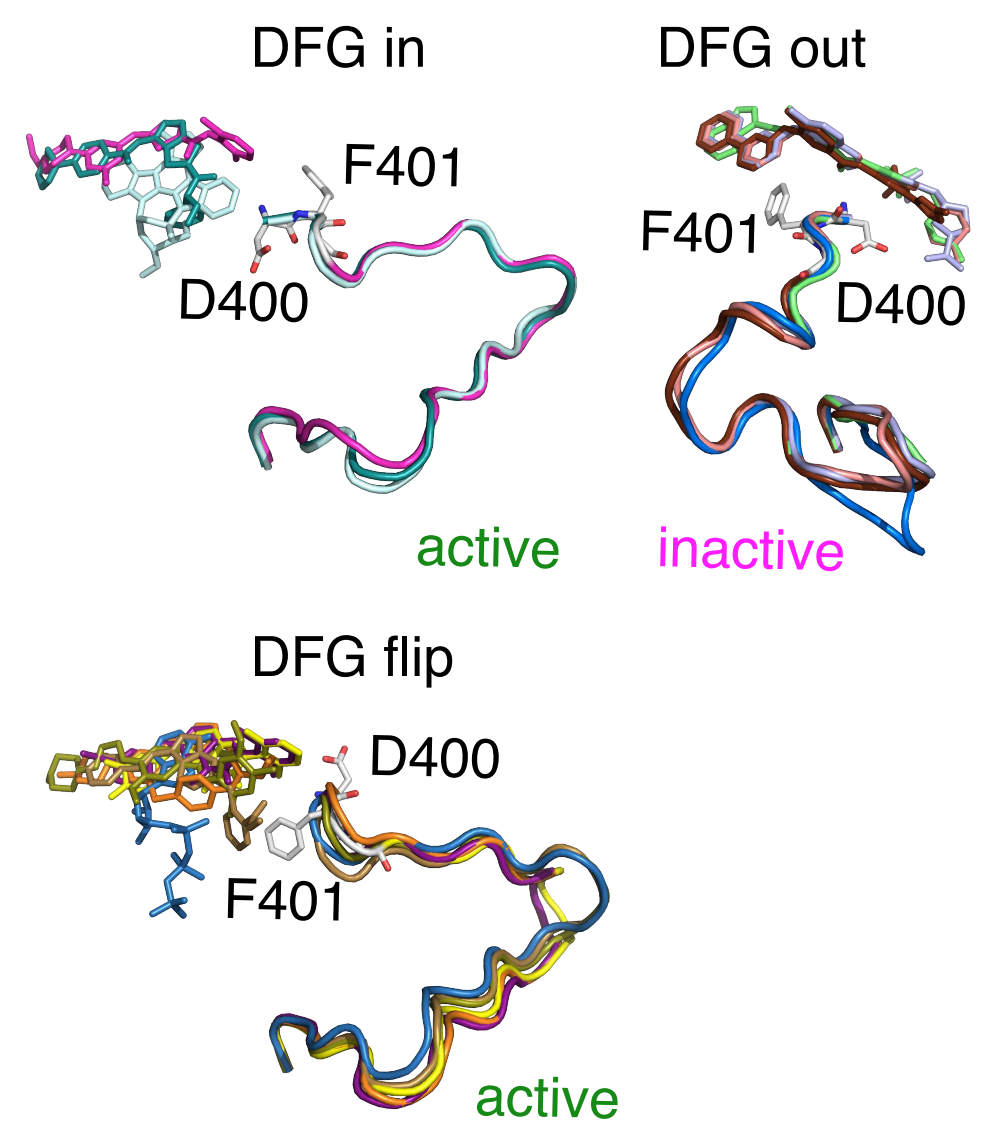




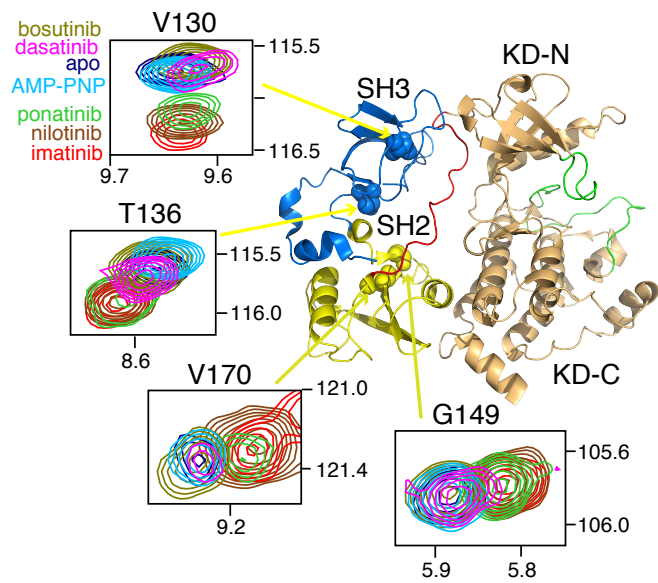

b

disassembled

assembled
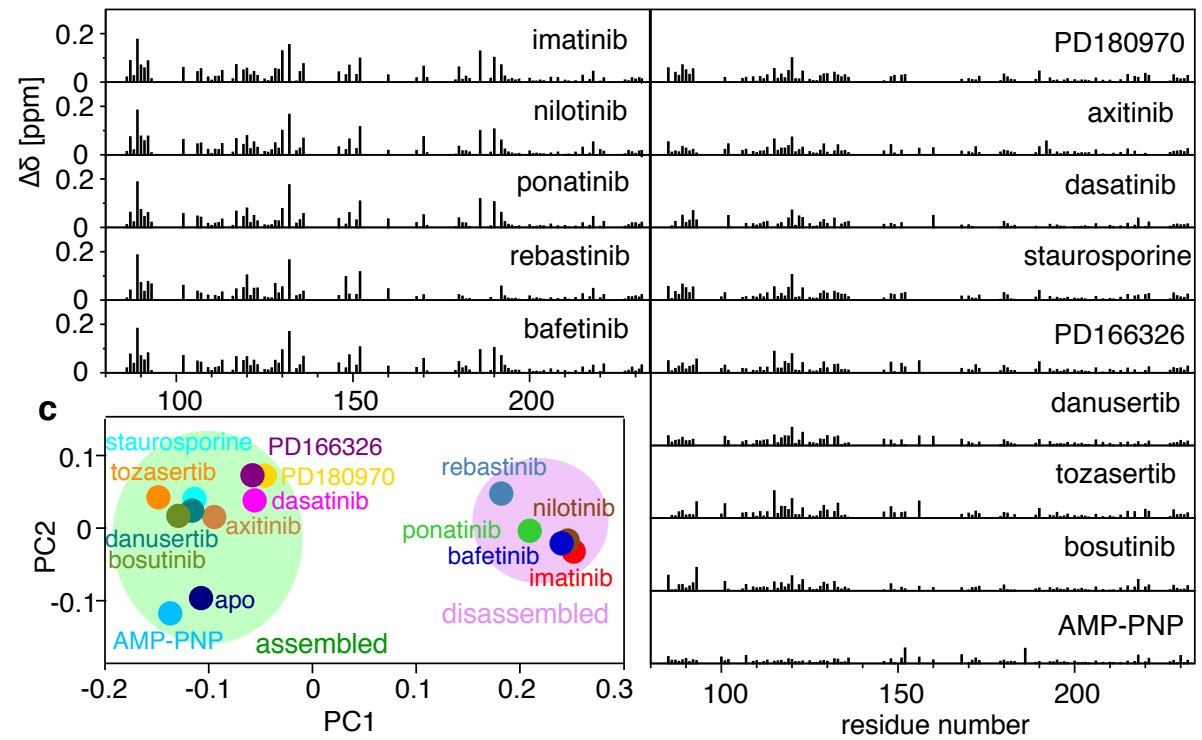

d
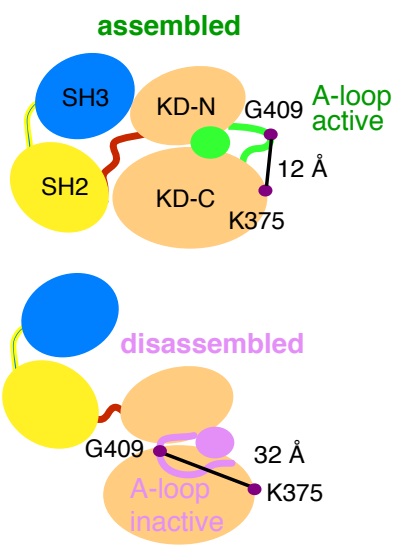

e

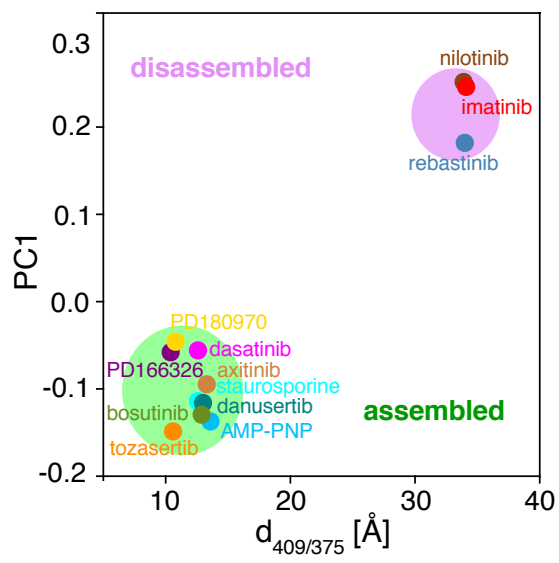

Fig 2. Sonti et al. 
a

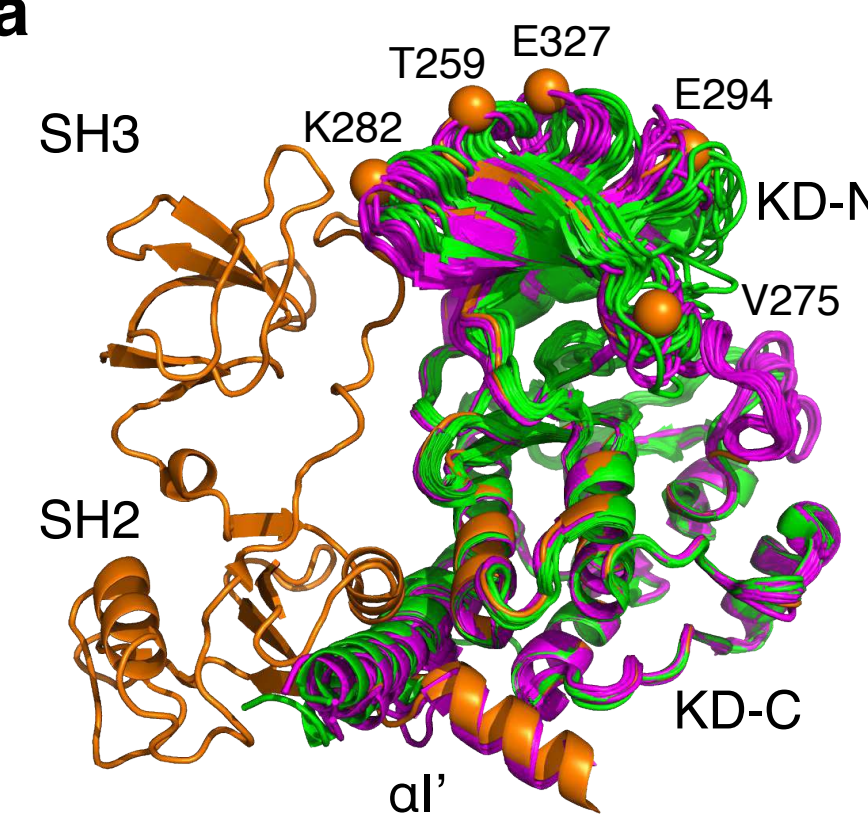

d

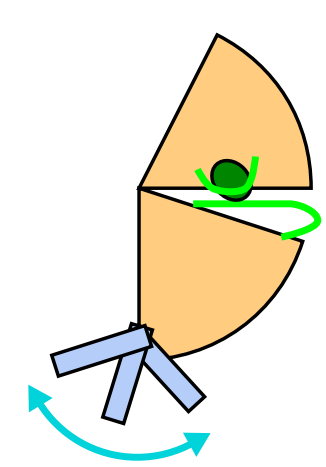

KD॰type I

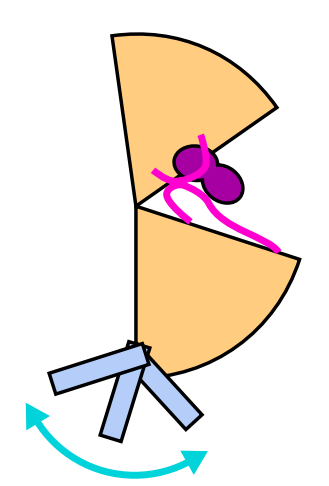

KD•type II b
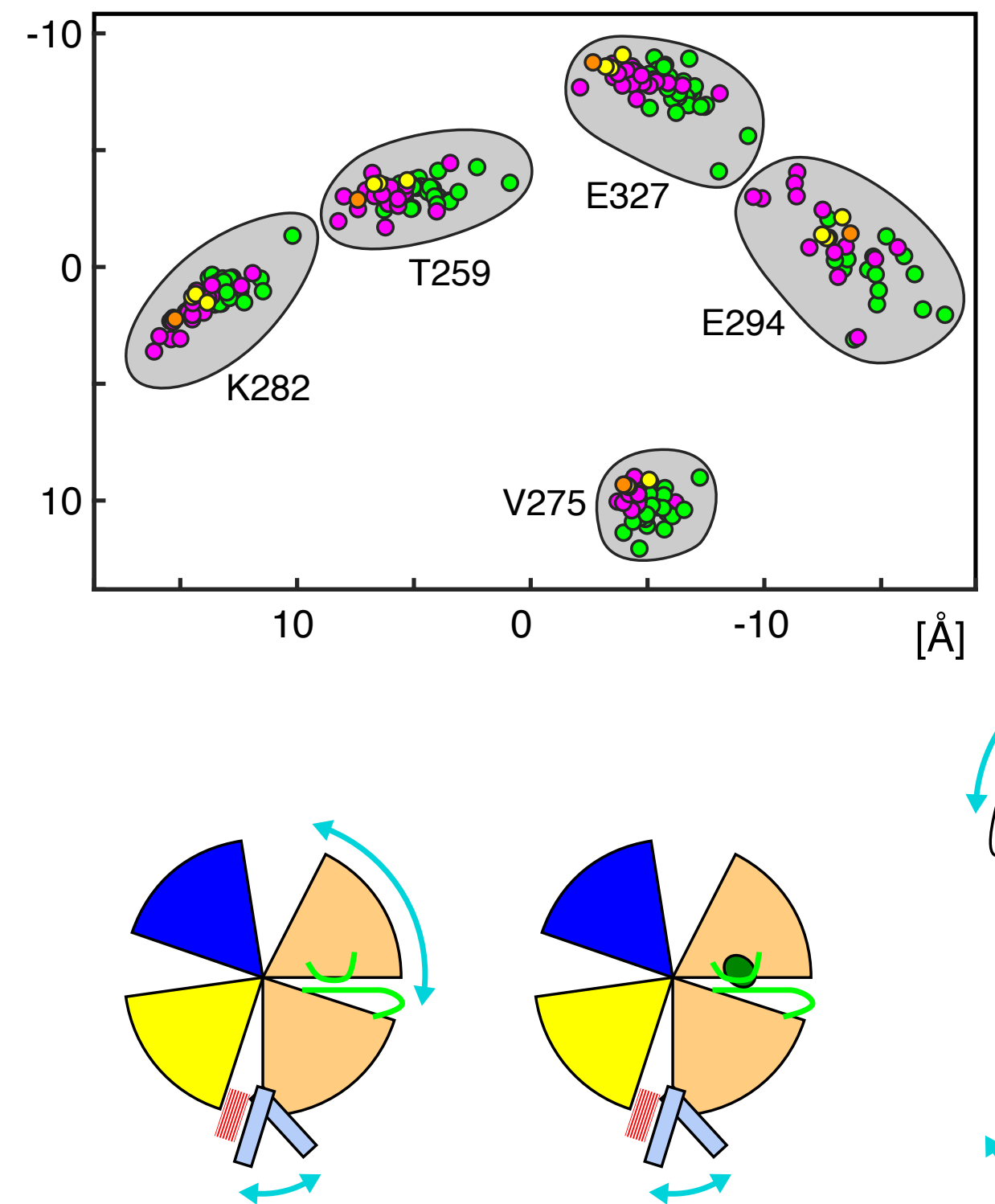

core apo

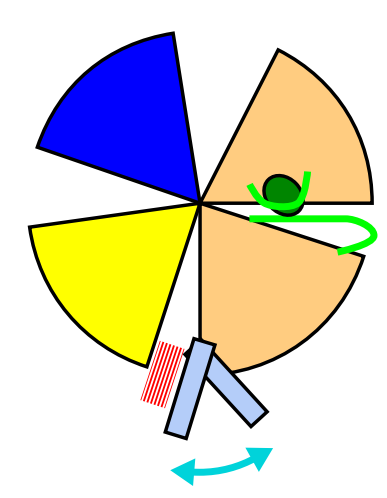

core•type I
C

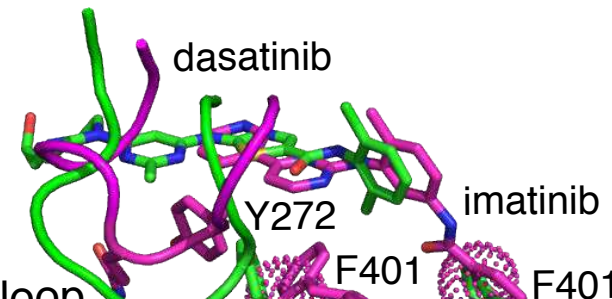

P-loop $\quad$ F401

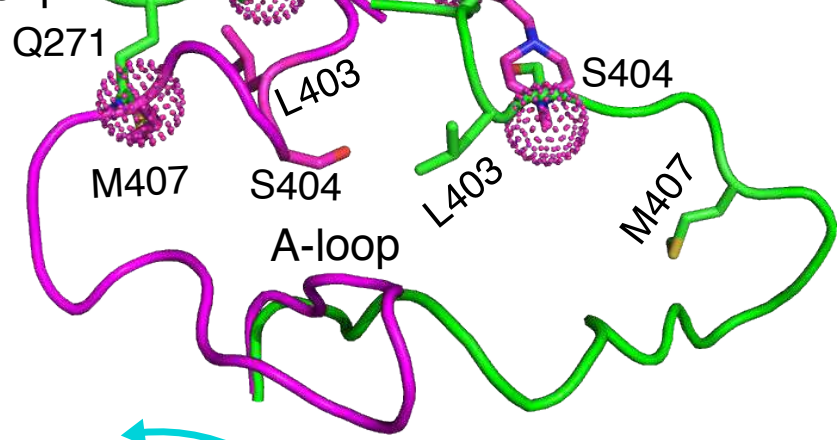

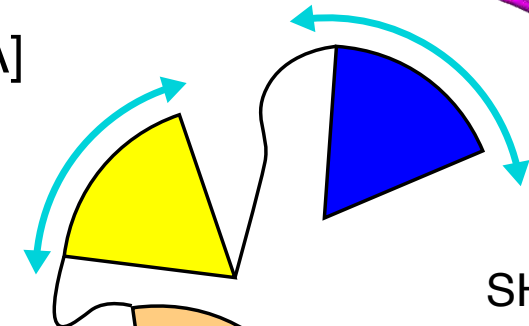

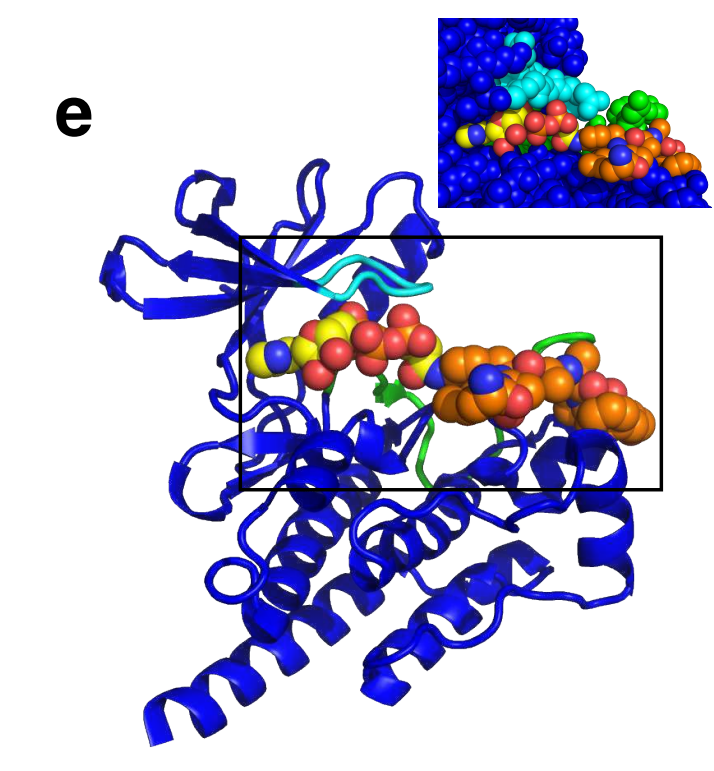

KD•substrate

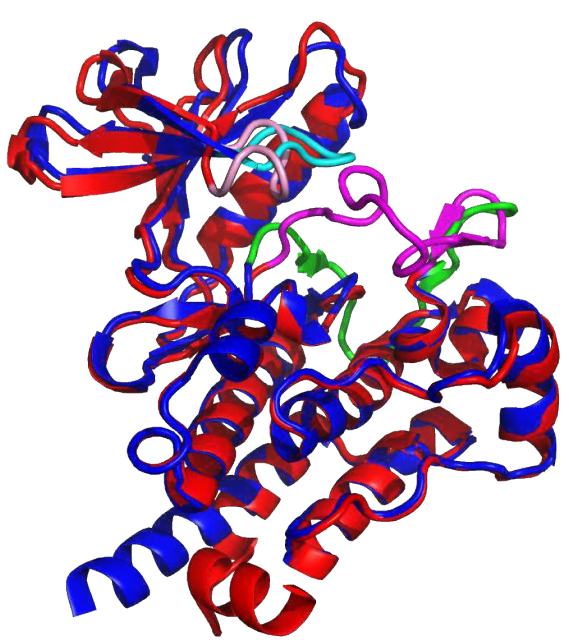

KD•type II

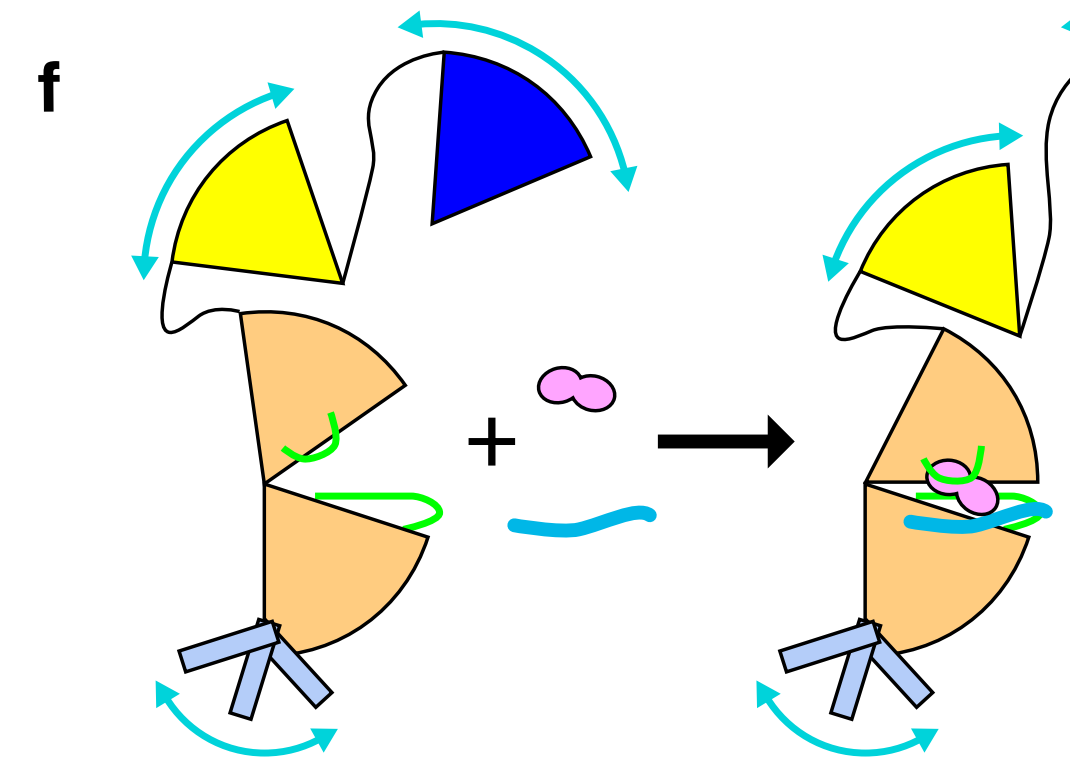

disassembled, active

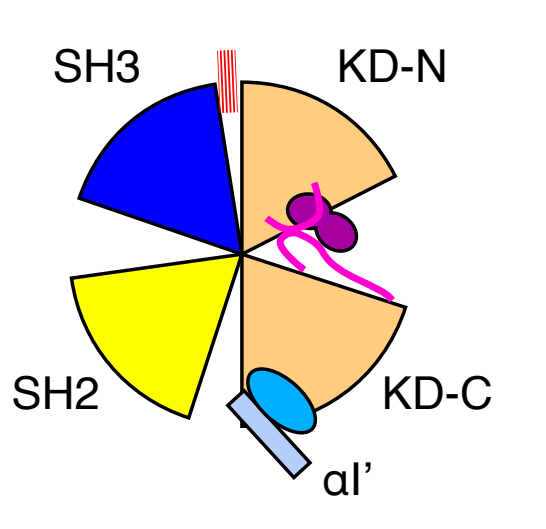

core•type II + allosteric 